\title{
Simulation de la variabilité de la photosynthèse hivernale du douglas (Pseudotsuga menziesii Mirb) dans les conditions climatiques françaises
}

\author{
E Choisnel 1*, V Jacq 1, JM Guehl 2, G Aussenac 2 \\ 1 Météorologie nationale, subdivision d'agrométérologie, 2 avenue Rapp, 75007 Paris; \\ 2 INRA, centre de Nancy, station de sylviculture et production, laboratoire de bioclimatologie- \\ écophysiologie, Champenoux, 54280 Seichamps, France
}

(Reçu le 01 août 1989; accepté le 02 juillet 1990)

\begin{abstract}
Résumé - Sur la base d'un modèle mathématique simple mis au point dans une étude antérieure (Guehl et al, 1985), une simulation du fonctionnement photosynthétique hivernal du douglas a été effectuée pour 96 stations du réseau de la Météorologie nationale française entre les années 1949 et 1985, permettant l'étude de la variabilité spatio-temporelle de la potentialité de photosynthèse hivernale du douglas en France. Contrairement aux régions boréales, continentales et de montagne, à hivers rigoureux, les zones de plaine en France se caractérisent globalement par des potentialités significatives d'assimilation carbonée en dehors de la période de croissance. Au nord-est d'une ligne passant par Paris et Lyon, ces potentialités sont faibles et présentent une forte variabilité interannuelle. Au sud-ouest de cette ligne, les potentialités sont plus élevées et plus stables dans le temps.
\end{abstract}

douglas / photosynthèse hivernale / modèle mathématique / simulation / variabilité spatiotemporelle

Summary - Simulation of the variability of winter photosynthesis of Douglas fir (Pseudotsuga menziesii Mirb) under French climatic conditions. By means of a mathematical model developed in a previous study (Guehl et al, 1985) a simulation of the winter photosynthetic functioning of Douglas fir was carried out between 1949 and 1985 on the basis of climatological data from 96 stations of the national meterological network in France, thus allowing the assessment of the geographic and time variability of the winter photosynthetic potentialities in France. Contrarily to regions with severe winters of higher latitude or altitude, or more continental climates, there are significant photosynthetic potentialities outside the growing period in the low elevation regions of France (fig 5). North-east of a line running through Paris and Lyon (figs 6, 7, 8), these potentialities are low and exhibit a high time variability (table II). South-west of that line the potentialities are higher and become more stable with time.

Douglas fir / winter photosynthesis / mathematical modelling / simulation / geographic and time variability

\footnotetext{
* Correspondance et tirés à part
} 


\section{INTRODUCTION}

L'utilisation raisonnée des espèces forestières pour les reboisements suppose une bonne connaissance du déterminisme environnemental et notamment climatique des processus concourant à la croissance.

Différentes études (Emmingham et Waring, 1977; Fry et Phillips, 1977; Guehl, 1985; Guehl et al, 1985) ont montré que les conifères de la zone tempérée sont capables de photosynthétiser de façon significative en dehors de la période de croissance végétative. Les photosynthétats résultant de l'assimilation hivernale de $\mathrm{CO}_{2}$ sont utilisés en partie pour le métabolisme énergétique et la croissance racinaire qui peut se poursuivre tout au long de l'hiver. Une autre partie de ces photosynthétats est stockée sous forme de glucides essentiellement dans les aiguilles (Senser et al, 1975; Ericsson, 1979) et le parenchyme cortical et xylémien des tiges et des racines (Krueger et Trappe, 1967; Webb, 1977; Ericsson et Persson, 1980), puis remobilisée au printemps au cours d'une phase de croissance racinaire intense et de la phase initiale de croissance aérienne (Krueger et Trappe, 1967; Webb, 1977; Ericsson, 1978, 1979; Ericsson et Persson, 1980).

Au plan plus global de l'étude de la production ligneuse, des études dendroclimatologiques (Becker, 1988) ont montré que les températures hivernales basses ont un effet dépressif sur la croissance en circonférence durant la saison de végétation suivante. On peut penser que ce phénomène est expliqué pour partie par des bilans d'assimilation hivernale faibles. Une autre partie de l'explication peut sans doute être attribuée à des dégâts liés au gel.

L'importance de l'assimilation carbonée hivernale, variable selon les espèces (Schwarz, 1971; Guehl, 1985; Guehl et al, 1985), est fortement modulée par les conditions thermiques et l'éclairement. En France, où les conditions climatiques sont géographiquement assez variables, on peut s'attendre à des potentialités de photosynthèse hivernale très différentes suivant les régions. Le fonctionnement photosynthétique hivernal a été effectivement étudié chez plusieurs espèces d'intérêt économique : Pseudotsuga menziesii, Abies alba, Picea abies, Cedrus atlantica, Cedrus libani. II a été mis en évidence que, dans la gamme des températures hivernales habituelles en France, la sensibilité aux basses températures est minimale pour les espèces ou provenances originaires de régions à hivers tempérés (Guehl, 1985; Guehl et al, 1985). Dans le cas du douglas (Pseudotsuga menziesii) il a été possible (Guehl et al, 1985) de mettre au point un modèle de simulation du fonctionnement photosynthétique hivernal, car la capacité photosynthétique est expliquée de façon simple par l'histoire thermique récente des rameaux. $\mathrm{Ce}$ modèle rend possible l'étude de la variabilité climatique de la potentialité de photosynthèse hivernale du douglas en France et permet d'en proposer une analyse statistique de variabilité dans l'espace et dans le temps.

La présente analyse a pour objectif de mettre en évidence, à l'échelle de la France, l'impact des conditions climatiques hivernales sur l'indice de production étudié ici, à savoir l'assimilation de $\mathrm{CO}_{2}$ d'un rameau de pleine lumière.

\section{MÉTHODES D'ÉTUDE}

\section{Description du modèle de fonctionnement photosynthétique hivernal}

Le modèle est basé sur des mesures menées en conditions environnementales contrôlées de laboratoire et portant sur : 
- l'évolution au cours de l'hiver de la capacité photosynthétique de rameaux de pleine lumière excisés prélevés sur des arbres d'un peuplement âgé de 22 ans (mesures hebdomadaires),

- les réponses instantanées du taux d'assimilation nette de $\mathrm{CO}_{2}(\mathrm{~A})$ à l'éclairement et à la température de l'air, et de la respiration à l'obscurité $\left(R_{0}\right)$ à la température de l'air.

Le modèle, dont la structure générale est présentée dans la figure 1 , donne accès au bilan d'assimilation carbonée d'un rameau de l'année supposé être placé en conditions de pleine lumière (partie supérieure du houppier). II fait intervenir de façon successive des limitations environnementales liées à l'histoire thermique des rameaux, à la température actuelle, à l'intensité du rayonnement solaire incident et à la durée du jour. Dans le tableau I on a fait apparaître les caractéristiques des différents sous- modèles prenant en compte ces étapes successives. On trouvera dans Guehl et al (1985) une discussion portant sur la justification des hypothèses utilisées pour la construction du modèle. L'hypothese de constance du rendement lumineux maximal $k_{2}$ (tableau I) en fonction de la température actuelle $\theta$ est confirmée par les travaux de Leverenz et Öquist (1987) mettant en évidence une quasi-constance de $k_{2}$ entre -2 et $+35^{\circ} \mathrm{C}$ pour Pinus silvestris. Les relations entre taux assimilation de $\mathrm{CO}_{2}(A)$ et éclairement et entre $A$ et $\theta$ utilisées dans le modèle sont donnés dans la figure 2.

\section{Mise en cuvre du modèle}

Le modèle de fonctionnement photosynthétique présenté ci-dessus peut être utilisé en prenant

Tableau I. Description des sous-modèles du schéma de la figure 1 et rappel des hypothèses utilisées. $T_{\text {moy } 1}$ moyenne arithmétique entre la température minimale de la nuit précédant la journée considérée et la température maximale du jour précédent; $R_{o \jmath}$, taux de respiration à l'obscurité diurne; RoN, taux de respiration nocturne. Pour la signification des autres symboles se reporter à la figure 1.

\begin{tabular}{|c|c|c|c|c|}
\hline $\begin{array}{l}\text { Sous- } \\
\text { modèle }\end{array}$ & $\begin{array}{l}\text { Variables } \\
\text { d'entrée }\end{array}$ & $\begin{array}{l}\text { Variables } \\
\text { de sortie }\end{array}$ & Equations & Hypothèses utilisées \\
\hline $\mathbf{a}$ & $T_{\text {moy } 1}$ & $A_{\max }$ & $A_{\max }=0,010 \mathrm{~T}_{\text {moy } 1}+0,108$ & $\begin{array}{l}\text { On impose une borne supérieure } \\
A_{\max }=0,264 \mathrm{mg} \cdot \mathrm{m}^{-2} \cdot \mathrm{s}^{-1}\end{array}$ \\
\hline b & $\begin{array}{l}\boldsymbol{A}_{\max } \text { et temp } \\
\text { moy horaire } \\
\text { diurne }(\theta)\end{array}$ & $A_{\text {sat }}$ & $\begin{array}{l}\text { Ajustement parabolique } \\
\text { par intervalles (cf fig } 2 \mathrm{~b} \text { ) }\end{array}$ & $\begin{array}{l}\text { Forme de la relation } A(\theta) \\
\text { invariable dans le temps }\end{array}$ \\
\hline $\begin{array}{l}c_{1} \\
c\end{array}$ & $\begin{array}{l}\text { Temp moy } \\
\text { horaire }(\theta)\end{array}$ & $R_{O J}$ & $R_{o J}=0,0048 \mathrm{e}^{0,0896 . \theta}$ & $\begin{array}{l}\text { Relation } R_{o}(\theta) \text { constante dans } \\
\text { le temps au cours de l'hiver }\end{array}$ \\
\hline$c_{2}$ & $\begin{array}{l}A_{\text {sat }}, R_{o J} \text { et } \\
\text { rayonnement } \\
\text { horaire } \\
(\mathrm{RH})\end{array}$ & A & $\begin{array}{l}A=k_{1} \operatorname{tgh}\left(\left(k_{2} / k_{1}\right) R_{H}\right)-R_{O J} \\
k_{1}: \text { valeur théorique } \\
\text { maximale de } A+R_{O J} \\
-) k_{1}=A_{\text {sat }}+R_{o J} \\
\text { si } A_{\text {sat }}+R_{O J}<0,139 \\
-) k_{1}=0,04 \mathrm{e}^{8,89}\left(A_{\text {sat }}+R_{O J}\right) \\
\text { si } A_{\text {sat }}+R_{O J} \geq 0,139 \\
k_{2}=1,6910^{-3} \mathrm{mg} \mathrm{CO}_{2}\end{array}$ & $\begin{array}{l}k_{2}, \text { pente à l'origine de la relation } \\
\text { assimilation-éclairement } \\
\text { constante en fonction de } \theta \text { et } \\
\text { de l'histoire thermique } \\
\text { des rameaux }\end{array}$ \\
\hline e & $\theta$ nocturne & $R_{O N}$ & $R_{O N}=0,0048 \mathrm{e}^{0,0896 \theta}$ & $\begin{array}{l}\text { Mêmes relations } R_{o}(\theta) \text { le jour } \\
\text { et la nuit }\end{array}$ \\
\hline
\end{tabular}


Histoire thermique

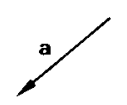

Capacité d' assimilation

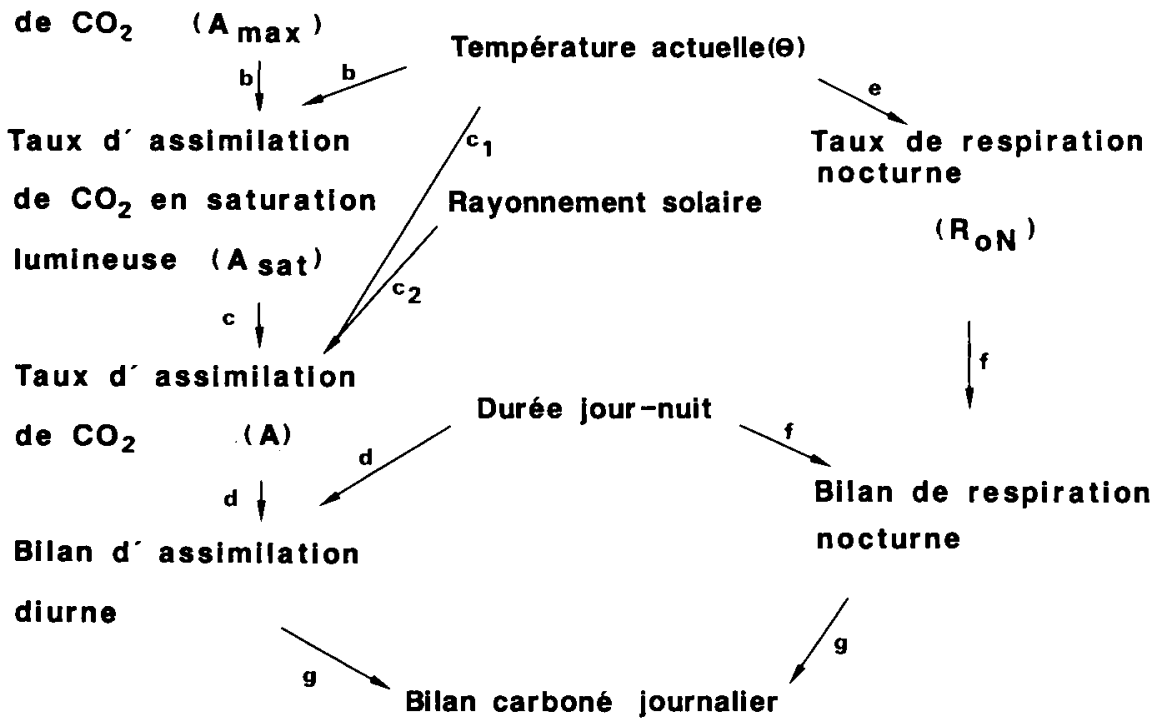

Fig 1. Structure du modèle utilisé pour l'estimation du bilan journalier d'assimilation carbonée. Les lettres a à $g$ représentent les différents sous-modèles qui sont décrits dans le tableau I sauf $d, f$ et $g$ qui correspondent à de simples opérations de sommation.

comme données d'entrée des données météorologiques couramment disponibles dans les stations du réseau synoptique couvrant le territoire français (fig 3). Il faut pour cela opérer une légère transformation des données de base pour les adapter au pas de temps (l'heure) choisi pour le modèle.

Des 4 variables successives calculées ( $A_{\max }$, $A_{\text {sat }}, A$ et $R_{0}$; voir tableau I), $A_{\max }$ est calculée une fois par jour et les trois autres sont calculées au pas de l'heure. Les valeurs horaires de température de l'air $(\theta(H))$ sont une combinaison linéaire du minimum et du maximum journaliers (Choisnel, 1977) :

$$
\theta(H)=\theta_{N}+\left(\theta_{X}-\theta_{N}\right) k(H)
$$

La fonction $k(H)$ dépend de la saison et la figure 4 en donne les courbes d'évolution nycthémérales pour les 4 saisons. $\theta_{N}$ et $\theta_{X}$ sont respectivement les températures minimale et maximale journalières.

Le rayonnement solaire global horaire $\left(R_{H}\right)$ est calculé en 2 temps (cf annexe) : on calcule d'abord sa valeur journalière par régression statistique, fonction de la durée d'insolation, ensuite ce total journalier est réparti heure par heure entre le lever et le coucher du soleil en séparant la partie directe et la partie diffuse et en supposant une nébulosité constante au cours de la journée. Les fonctions de répartition dépendent d'une seule variable : la hauteur angulaire du soleil au-dessus de l'horizon. l'heure de début de l'ensoleillement $\left(H_{\nu}\right)$ correspond à la 

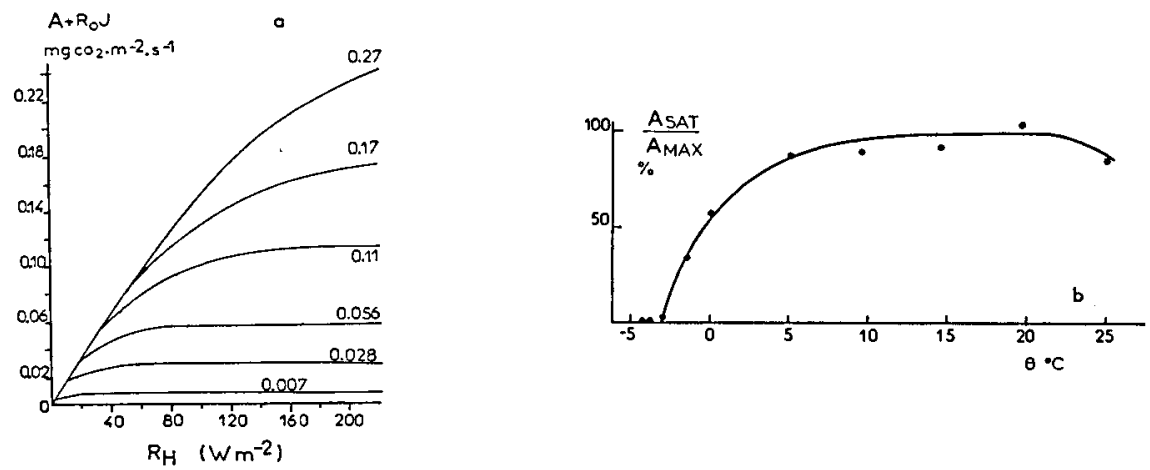

Fig 2 (a) Relations entre assimilation de $\mathrm{CO}_{2}\left(A+R_{0}\right)$ et rayonnement global horaire $(R H)$ pour différentes valeurs maximales théoriques de $\left(A+R_{0}\right)$ indiquées sur la figure; (b) relation entre assimilation de $\mathrm{CO}_{2}$ (rapport $\mathrm{A}_{\text {sat }} / \mathrm{A}_{\max }$ ) et température $(\theta)$. Cette dernière relation peut être ajustée par les équations suivantes :

$\theta<-3$,

$-3 \leq \theta<2,5, A_{\text {sat }} / A_{\max }=0$

湖 ${ }^{\circ}, \quad A_{\text {sat }} / A_{\max }=0,54+0,121 \theta-0,019 \theta^{2}+0,0023 \theta^{3}$

$0^{\circ} \mathrm{C}, A_{\text {sat }} / A_{\max }=0,50+0,112 \theta-0,010 \theta^{2}+0,0004 \theta^{3}$

$\theta \geq 10,0^{\circ} \mathrm{C}, \quad A_{\text {sal }} / A_{\max }=1,09-0,038 \theta+0,0034 \theta^{2}-0,0009 \theta^{3}$

première heure entière après le lever du soleil et l'heure de fin d'ensoleillement $\left(H_{\partial}\right)$ est la dernière heure entière précédant le coucher du soleil.

$A_{s a t}$ et $A$ sont d'abord cumulées sur les $h$ de jour, $R_{0}$ est cumulée sur les $\mathrm{h}$ de nuit. Ensuite les fonctions $A_{\max }, A_{\text {sat }} A$ et $A-R_{0}$ sont cumulées au pas de temps décadaire pour constituer le fichier des résultats à partir duquel est effectuée l'analyse statistique de variabilité spatiotemporelle présentée ci-après. Pour cette analyse ont été prises en compte les séries climatiques de 96 stations du réseau synoptique couvrant l'ensemble du territoire français métropolitain sur une période s'étendant du $1^{\mathrm{er}}$ octobre 1949 au 31 mars 1986.

\section{RÉSULTATS}

\section{Évolution de la capacité photosynthétique au cours de I'hiver}

La variabilité temporelle de la capacité photosynthétique pour une station donnée est représentée sous la forme de graphiques (figs $5 a$ et b) qui donnent l'évolution de l'assimilation carbonée pour la période du 1 er octobre au 31 mars et indiquent, pour chaque décade, les valeurs de la médiane, des premier et quatrième quintiles, du minimum et du maximum. Ces graphiques permettent à la fois d'évaluer la variabilité de l'assimilation de $\mathrm{CO}_{2}$ en un lieu et une décade donnés et de comparer les décades entre elles. Seule la courbe reliant les valeurs médianes peut être assimilée au profil d'un hiver normal. Les courbes reliant les valeurs du minimum et du maximum sont des courbes enveloppes mais ne correspondent pas au profil d'un hiver particulier.

Dans la figure $5 a$ on a donné en exemple les graphiques de variabilité pour les stations de Nancy, Rostrenen, Limoges et Pau, représentatives des gradients climatiques est-ouest et nord-sud. Pour Nancy la valeur de $20 \mathrm{~g} \mathrm{CO}_{2} \cdot \mathrm{m}^{-2}$. décade $^{-1}$ n'est pas dépassée en valeur 


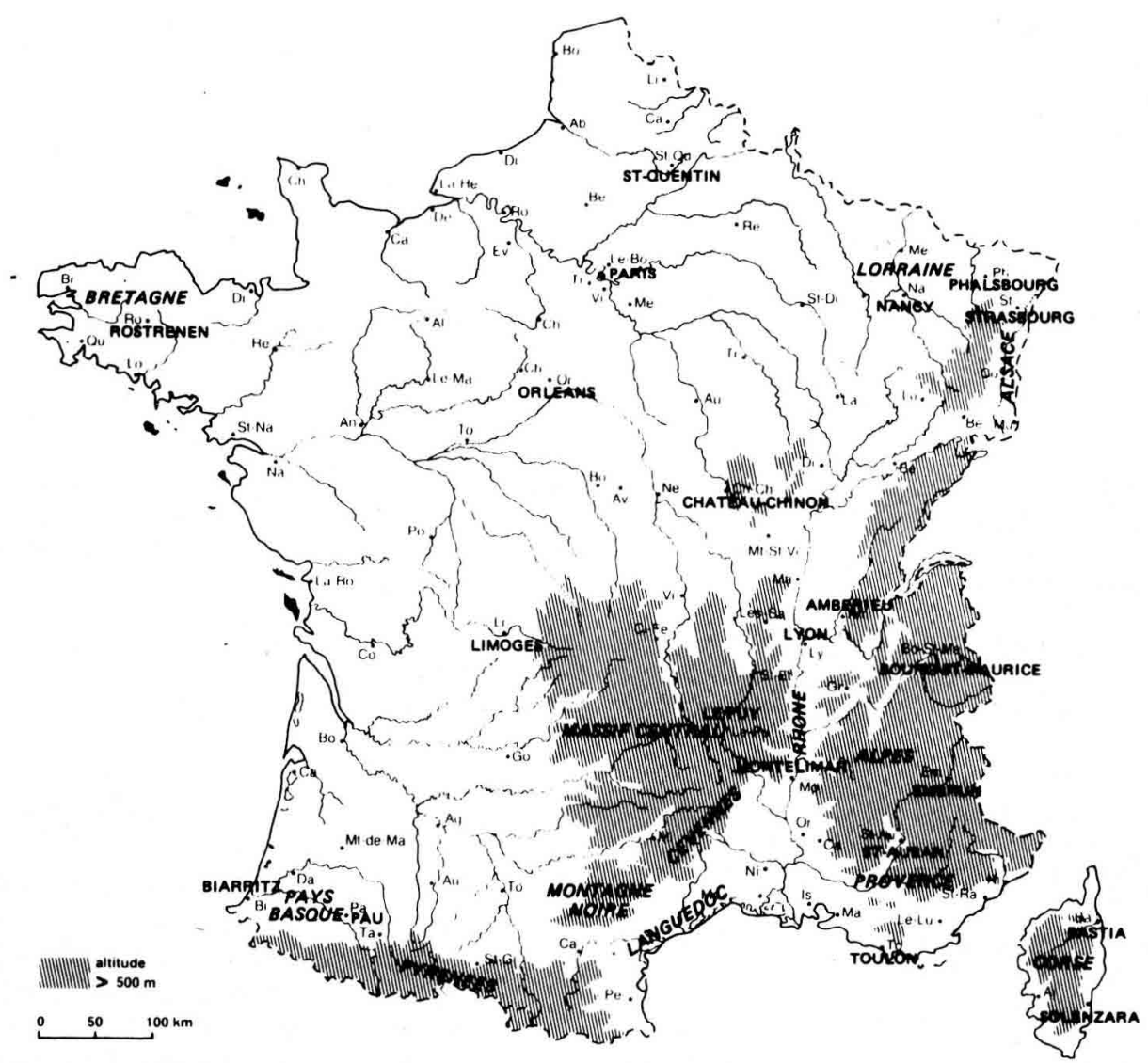

Fig 3. Repérage géographique (identification par des abréviations) des stations du réseau de la Météorologie nationale utilisées pour la simulation du fonctionnement photosynthétique hivernal du douglas en France. Les régions et stations auxquelles il est explicitement fait référence dans l'étude sont également indiquées sur la figure en toutes lettres.

médiane en décembre et janvier. La production photosynthétique peut être quasiment égale à zéro pour toute décade considérée individuellement de décembre à février. Pour une latitude similaire à Nancy $\left(48^{\circ} 1 \mathrm{~N}\right)$ on note à Rostrenen (climat océanique), des valeurs médianes supérieures à $20 \mathrm{~g} \mathrm{CO}_{2} \bullet \mathrm{m}^{-2} \cdot$ décade $^{-1}$ pour toute la période. Les valeurs des paramètres de dispersion sont très resserrées autour de la médiane, à l'exception de la valeur du minimum qui peut descendre en dessous de la valeur 10 en janvier et février. Des températures extrêmement basses en troisième décade de février 1986 induisent une valeur minimale singulière. La station de Limoges plus méridionale $\left(45^{\circ} 5 \mathrm{~N}\right)$ présente des valeurs de la médiane et du premier quintile proches de celles de Rostrenen pour toute décade de la période mais offre de meilleures possibilités photosynthétiques en cas de période 


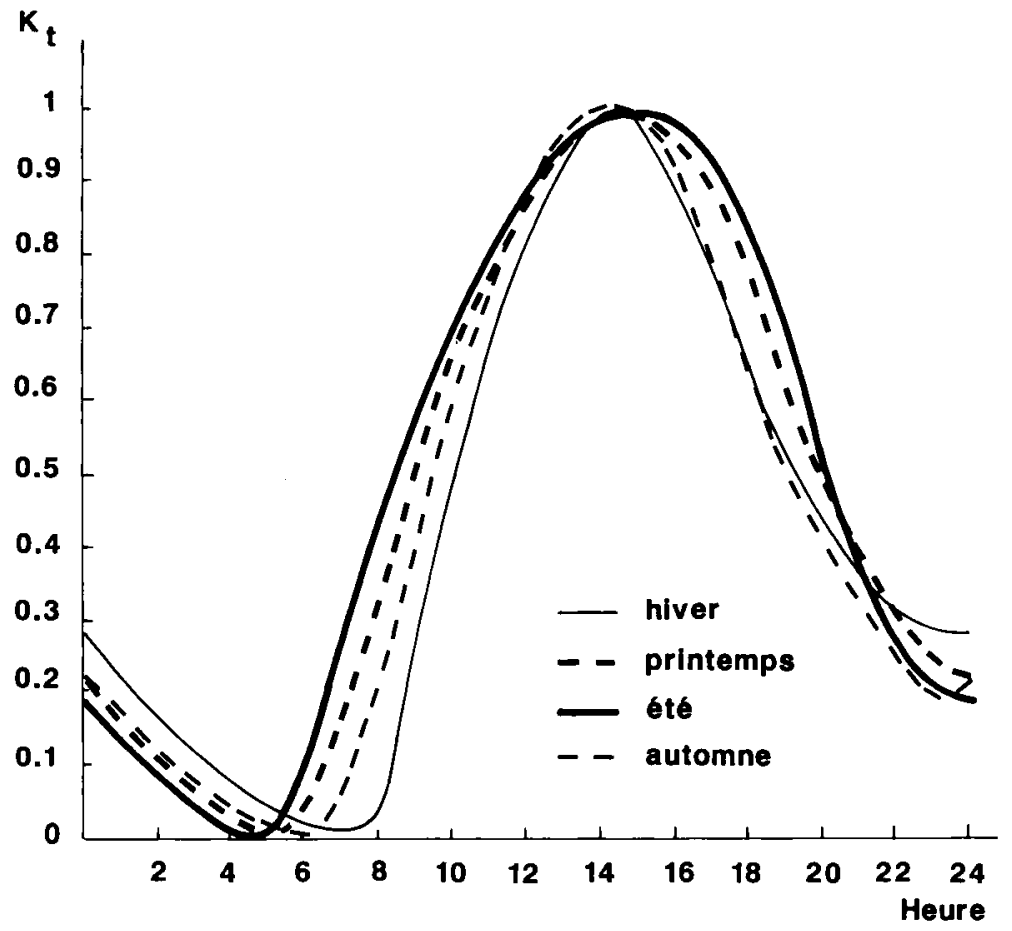

Fig 4. Variations nycthémérales du coefficient $k(H)$ pour les différentes saisons.

douce (cf valeurs du quatrième quintile et de la médiane). Enfin à Pau, de novembre à mars, les valeurs médianes sont supérieures d'environ $9 \mathrm{~g} \mathrm{CO}_{2} \cdot \mathrm{m}^{-2} \cdot$ décade $^{-1}$ à celles de Rostrenen. La variabilité de part et d'autre de la médiane est plus forte qu'à Rostrenen, mais le minimum ne descend jamais en dessous de la valeur $11,0 \mathrm{~g}$ $\mathrm{CO}_{2} \cdot \mathrm{m}^{-2}$.

Dans la figure $5 b$ on a regroupé quatre stations particulières, non représentatives d'une grande région, mais correspondant à des environnements de pourtour montagneux intéressants du fait de la présence de zones forestières limitrophes. II s'agit des stations de Château-Chinon, Ambérieu, Bourg-St-Maurice et Embrun. A Château-Chinon, on observe en février, une augmentation notable de la variabilité interannuelle par rapport à celle des mois précédents. Dans les Alpes, la comparaison entre Ambérieu $(253 \mathrm{~m})$ et Bourg-StMaurice $(868 \mathrm{~m})$ fait apparaître de décembre à mars, du fait de l'augmentation d'altitude, une baisse d'environ $5,5 \mathrm{~g}$ $\mathrm{CO}_{2} \cdot \mathrm{m}^{-2} \cdot$ décade $^{-1}$. La station d'Embrun à $876 \mathrm{~m}\left(44^{\circ} 30 \mathrm{~N}\right)$ de même altitude que Bourg-St-Maurice $\left(45^{\circ} 40 \mathrm{~N}\right)$ surpasse cette dernière station en valeur médiane d'environ $4,5 \mathrm{~g} \mathrm{CO}_{2} \cdot \mathrm{m}^{-2}$.décade ${ }^{-1}$.

\section{Cartographie des potentialités d'assimi- lation carbonée hivernale en France}

Une cartographie de la valeur médiane du bilan d'assimilation carbonée est présen- 

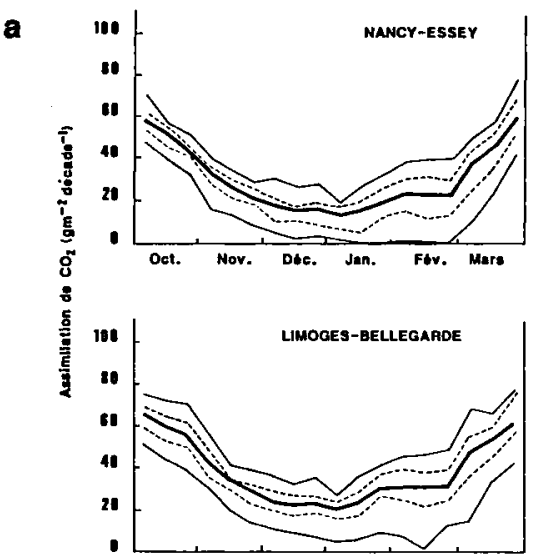

b
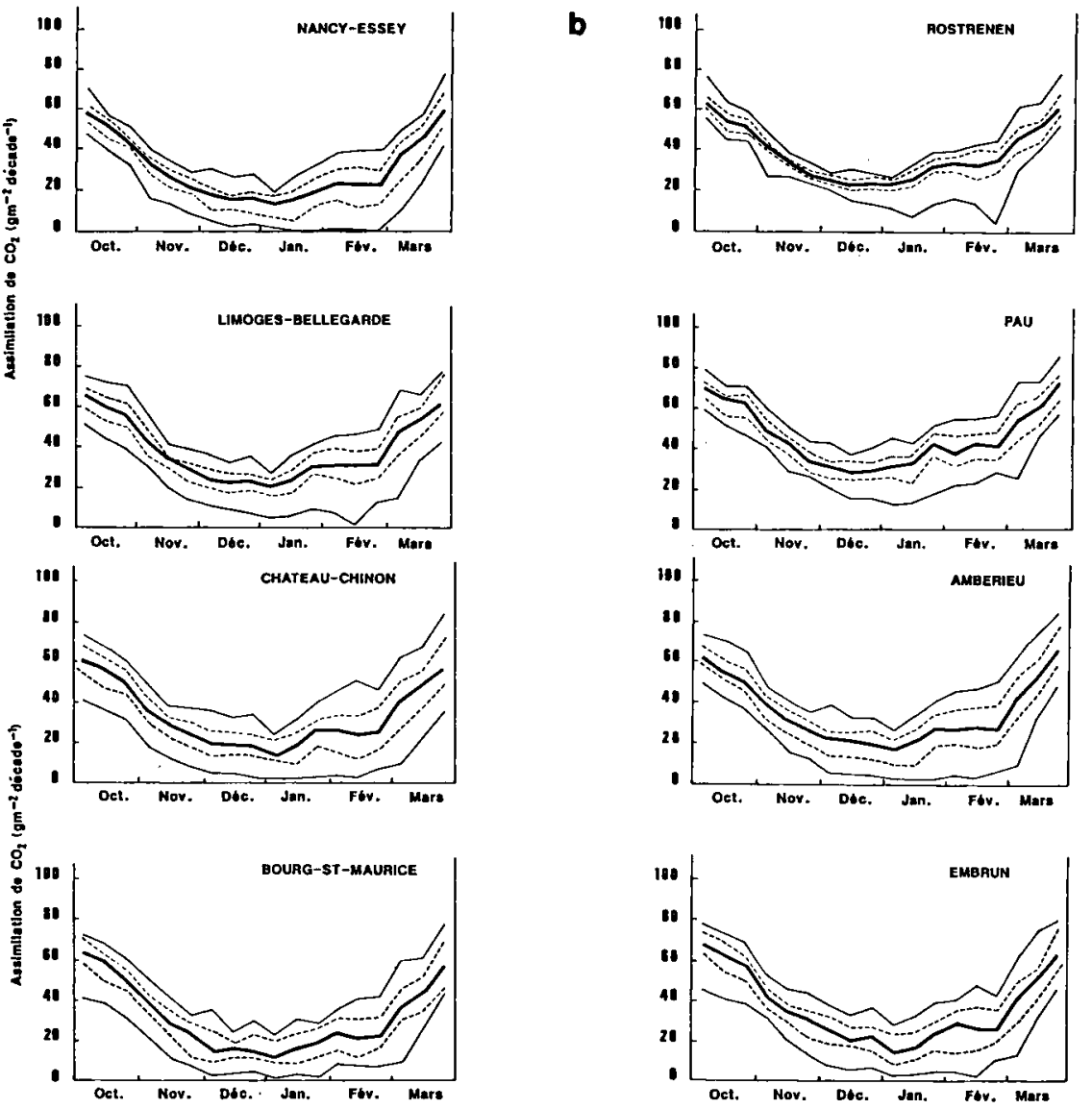

Fig $5 a$ et b. Simulation de l'évolution du bilan décadaire d'assimilation carbonée pour la période du 1 er octobre au 31 mars pour différentes stations du réseau de la Météorologie nationale. Les différentes courbes représentent respectivement du bas vers le haut les valeurs du minimum, du premier quintile, de la médiane (trait plein), du quatrième quintile et du maximum.

tée dans la figure 6 . Sur les figures 7 et 8 on a cartographié les valeurs correspondant à des hivers exceptionnels (19621963 pour les valeurs minimales de température et 1960-1961 pour les valeurs maximales). Pour compléter cette présentation cartographique des résultats, on a indiqué dans le tableau II les valeurs médianes, les valeurs des premier et quatrième quintile et les extrêmes pour 9 stations types.
On observe sur la figure 6 une décroissance, partant d'un maximum (plus de $800 \mathrm{~g} \mathrm{CO}_{2} \cdot \mathrm{m}^{-2}$ ) dans le Pays-Basque à un minimum (inférieur à $550 \mathrm{~g} \mathrm{CO}_{2} \cdot \mathrm{m}^{-2}$ ) en Lorraine et dans le Nord de l'Alsace. Le Massif armoricain constitue un minimum secondaire avec moins de $650 \mathrm{~g} \mathrm{CO}_{2} \cdot \mathrm{m}^{-2}$ à Rostrenen. La valeur minimale est observée à Phalsbourg avec $500 \mathrm{~g} \mathrm{CO}_{2} \cdot \mathrm{m}^{-2}$. Dans le sud-est de la France on observe un gradient important dans la vallée du 


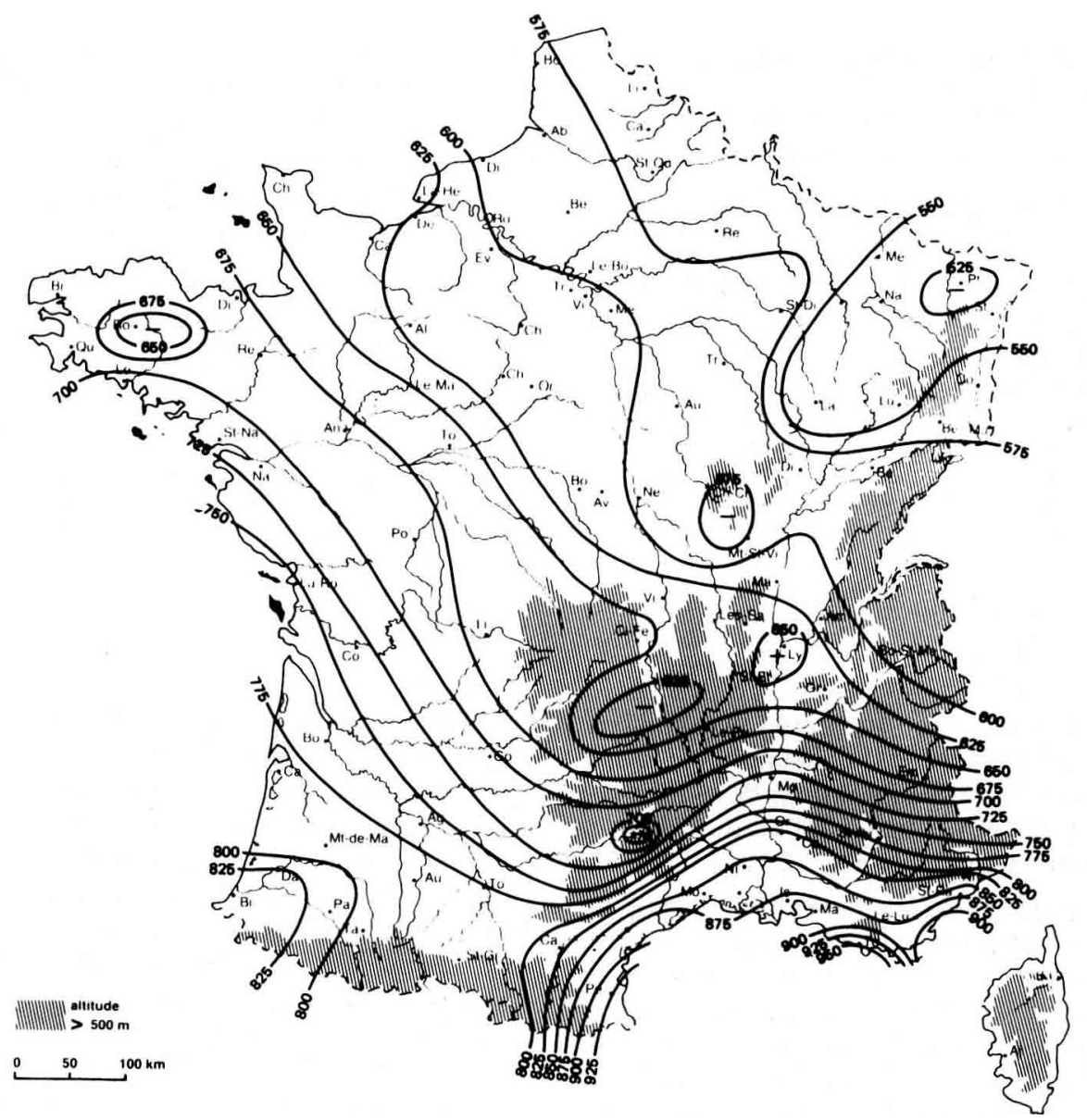

Fig 6. Variabilité géographique de la valeur médiane du bilan hivernal d'assimilation carbonée (période du $1^{\text {er }}$ octobre au 31 mars) exprimé en $\mathrm{g} \mathrm{CO}_{2} \cdot \mathrm{m}^{-2}$. Les zones hachurées correspondent aux zones d'altitude supérieure à $500 \mathrm{~m}$ pour lesquelles le tracé des lignes d'isovaleurs n'est pas fiable.

Rhône en amont de Montélimar. Les valeurs les plus élevées pour le continent sont observées dans la région de Toulon avec plus de $950 \mathrm{~g} \mathrm{CO}_{2} \cdot \mathrm{m}^{-2}$, et sur le littoral est de la Corse avec $975 \mathrm{~g} \mathrm{CO}_{2} \cdot \mathrm{m}^{-2}$ à Bastia et $991 \mathrm{~g} \mathrm{CO}_{2} \cdot \mathrm{m}^{-2}$ à Solenzara.

La cartographie du potentiel photosynthétique au cours de l'hiver froid 19621963 (fig 7) donne une configuration des gradients spatiaux semblable au cas de la valeur médiane : diminution selon un axe sud-ouest/nord-est et gradient perpendiculaire à la côte en Provence-Côte d'Azur et Languedoc-Roussillon. L'écart à la valeur médiane $\left(50 \mathrm{~g} \mathrm{CO}_{2} \cdot \mathrm{m}^{-2}\right)$ est faible pour le Pays basque et la Région provençale alors qu'il est maximal $\left(100 \mathrm{~g} \mathrm{CO}_{2} \cdot \mathrm{m}^{-2}\right.$ en Lorraine et au nord de Strasbourg). A l'inverse, pour l'hiver 1960-1961 exceptionnel- 
Tableau H. Caractéristiques statistiques interannuelles du bilan d'assimilation carbonée hivernal (période du 1 er octobre au 31 mars) pour 9 stations représentatives du réseau de la Météorologie nationale. Statistiques sur la série climatique 1949-1985.

\begin{tabular}{|c|c|c|c|c|c|c|c|}
\hline Station & Minimum & Q1 & $\begin{array}{c}\text { Médiane } \\
\left(\mathrm{g} \mathrm{CO}_{2} \cdot m^{-2}\right)\end{array}$ & Q4 & Maximum & $\begin{array}{c}(\operatorname{Max}-M i n) / M e ́ d \\
(\%)\end{array}$ & $\begin{array}{l}\text { Ecart type } \\
\left(\mathrm{gCO}_{2} \cdot \mathrm{m}^{-2}\right)\end{array}$ \\
\hline Nancy & 451 & 507 & 526 & 584 & 613 & 31 & 40 \\
\hline St-Quentin & 475 & 529 & 559 & 593 & 625 & 27 & 35 \\
\hline Château-Chinon & 496 & 518 & 568 & 599 & 650 & 27 & 40 \\
\hline Le Puy & 511 & 539 & 598 & 632 & 672 & 27 & 43 \\
\hline Orléans & 516 & 578 & 616 & 645 & 684 & 27 & 37 \\
\hline Rostrenen & 564 & 609 & 640 & 661 & 694 & 20 & 30 \\
\hline Limoges & 604 & 635 & 669 & 687 & 719 & 17 & 28 \\
\hline St-Auban & 671 & 751 & 779 & 808 & 821 & 19 & 32 \\
\hline Pau & 719 & 783 & 804 & 830 & 853 & 17 & 32 \\
\hline
\end{tabular}

lement doux (fig 8), apparaît une diminution du gradient sud-ouest/nord-est avec un écart Biarritz-Phalsbourg de l'ordre de $280 \mathrm{~g} \mathrm{CO}_{2} \cdot \mathrm{m}^{-2}$ alors qu'il était de $360 \mathrm{~g}$ $\mathrm{CO}_{2} \cdot \mathrm{m}^{-2}$ pour l'hiver 1962-63 (fig 7).

Les données du tableau II montrent que la variabilité interannuelle du bilan d'assimilation hivernal est élevée pour les stations de faible valeur médiane (Nancy, Château-Chinon, St-Quentin, Le Puy et Orléans), cependant que la variabilité est plus réduite pour les stations de valeurs médianes élevées (Limoges, St-Auban, Pau).

\section{DISCUSSION ET CONCLUSIONS}

Les résultats obtenus ici grâce à la simulation montrent qu'il existe en France des potentialités d'assimilation carbonée hivernale significatives, contrairement aux régions à hivers rigoureux, boréales (Lundmark et al, 1988), continentales (Zelawski et Kucharska, 1967) ou de montagne (Pisek et Kemnitzer, 1968) où l'activité photosynthétique hivernale est quasi nulle.
En relation avec les gradients climatiques de continentalité et de septentrionalité, il existe en France une variabilité géographique importante de l'activité photosynthétique hivernale chez le douglas. Pour les régions d'utilisation possible du douglas, les zones à capacité de photosynthèse hivernale élevée sont les suivantes dans l'ordre décroissant : Corse, Pays basque, Pyrénées-Orientales, Montagne Noire et Cévennes, moitié Sud du Massif alpin, bordure atlantique, Bretagne, bordure Ouest du Massif Central. Ces zones sont aussi celles où la variabilité interannuelle est la plus faible. Pour cet ensemble de régions, la part de l'assimilation photosynthétique hivernale représente une partie significative du bilan annuel, d'autant plus que certaines d'entre elles (bordure atlantique et Bretagne sauf intérieur, Sud-Ouest du Massif Central, Préalpes de Provence, Pyrériées-Orientales) se caractérisent par l'existence de déficits hydriques estivaux importants limitant la photosynthèse. D'ailleurs dans certaines zones océaniques à étés secs de l'aire naturelle du douglas, la part hivernale de l'assimilation carbonée annuelle peut atteindre 30-55\% 


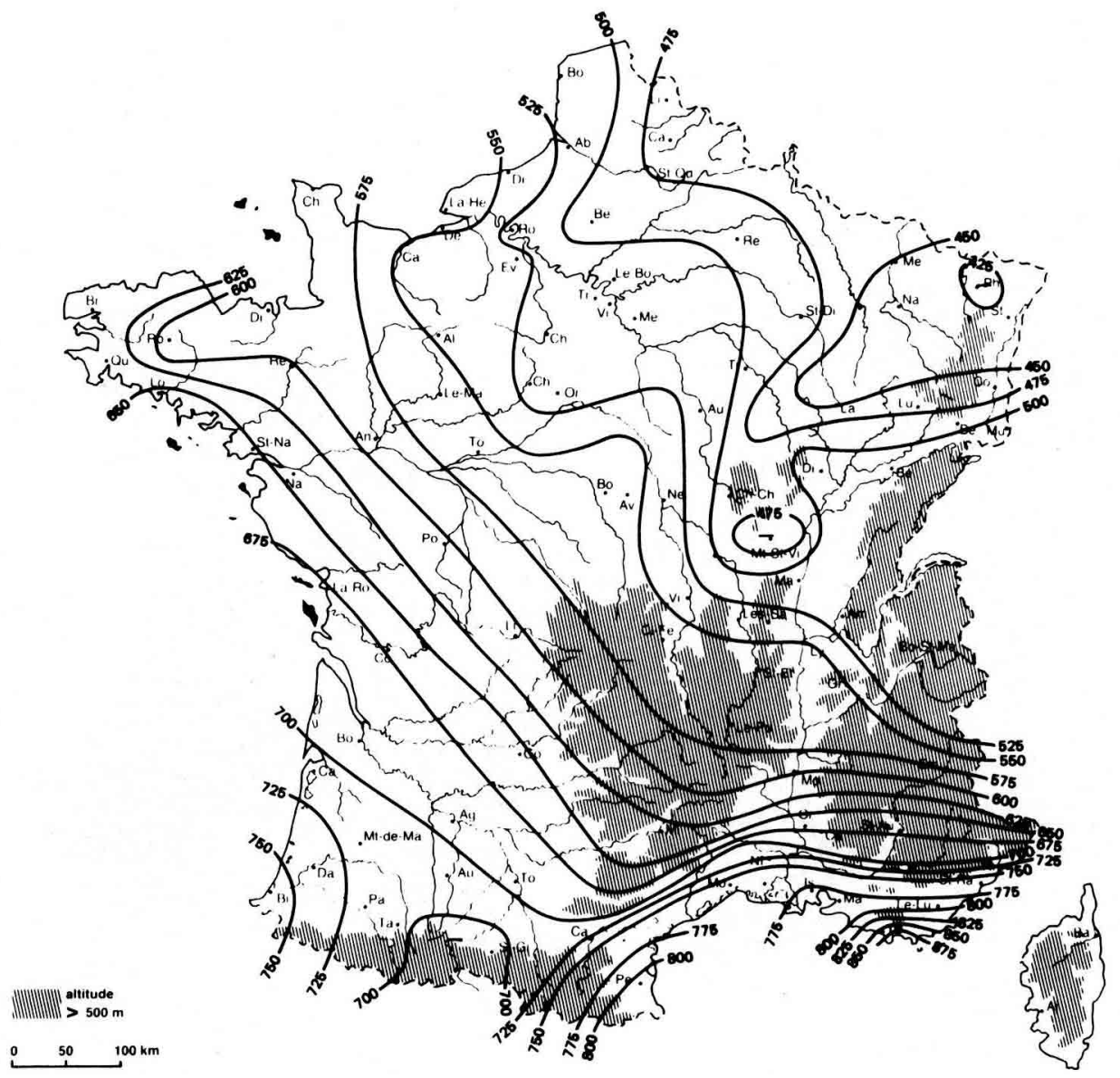

Fig 7. Variabilité géographique de la valeur du bilan d'assimilation carbonée exprimé en $\mathrm{g}\left(\mathrm{CO}_{2}\right) \cdot \mathrm{m}^{-2}$ sur la période du 1 octobre 1962 au 31 mars 1963.

(Emmingham et Waring, 1977). Au nordest d'une ligne passant par Paris et Lyon, les potentialités de photosynthèse hivernale sont nettement plus faibles (fig 6,7 et 8) et plus variables (tableau II) avec, pour certaines années, une production photosynthétique décadaire nulle de fin novembre à fin février (fig 5). En raison du manque de données climatologiques, it n'a pas été possible, sauf pour de rares cas, d'effectuer ces calculs pour les zones fo- restières montagnardes et d'étudier l'importance des gradients altitudinaux suivant les régions.

L'augmentation actuelle de la concentration atmosphérique en $\mathrm{CO}_{2}$, qui aura probablement pour conséquence une augmentation des températures hivernales en zone tempérée (Dickinson, 1986), devrait avoir pour conséquence une stimulation de l'activité photosynthétique hivernale des conifères. Dans ce contexte, le type de 


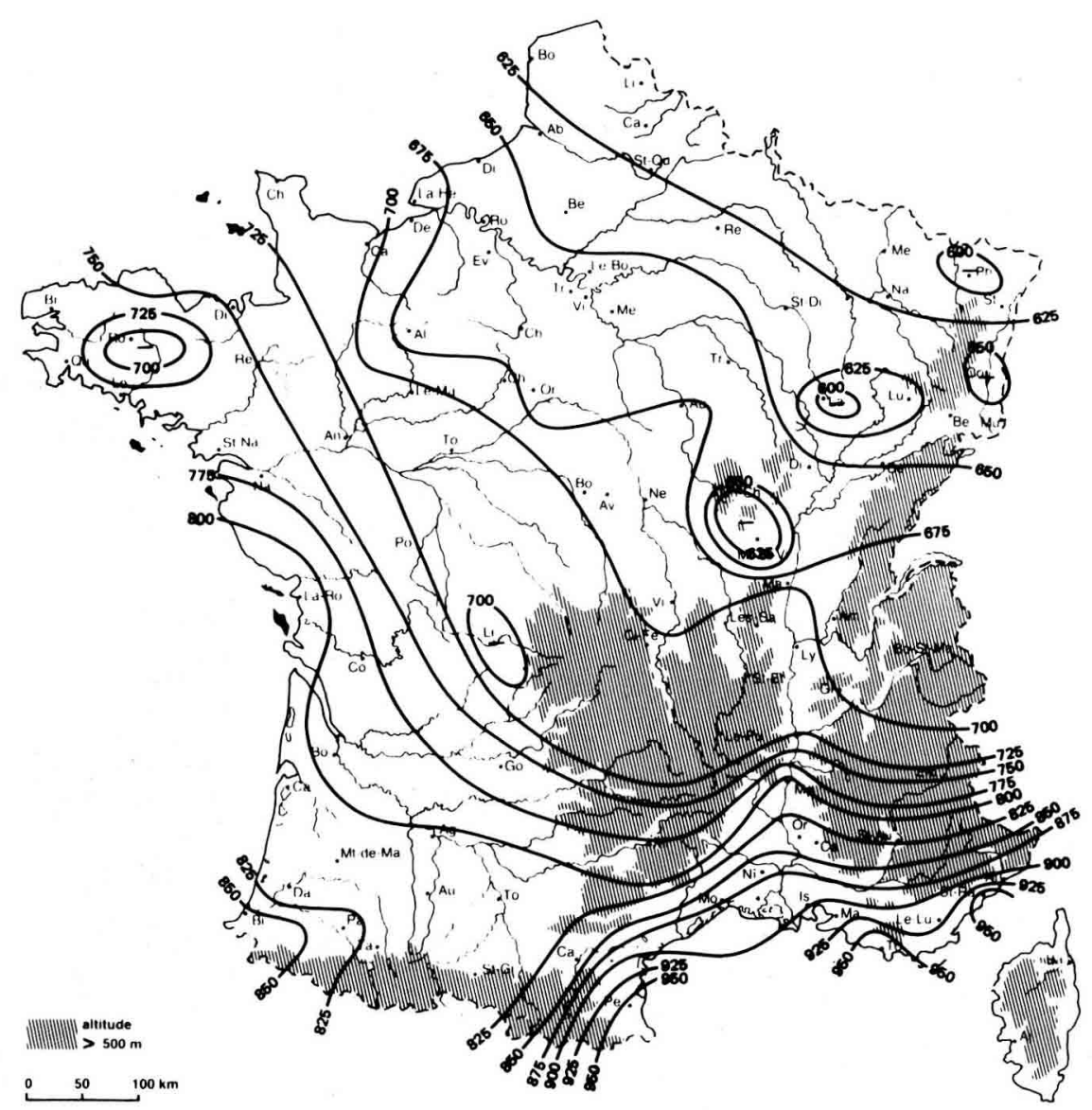

Fig 8. Variabilité géographique de la valeur du bilan d'assimilation carbonée exprimé en $\mathrm{g}\left(\mathrm{CO}_{2}\right) \cdot \mathrm{m}^{-2}$ sur la période du 1 octobre 1960 au 31 mars 1961.

modélisation utilisé dans la présente étude, est susceptible dans l'avenir, à partir des informations fournies par les modèles climatiques globaux, d'apporter des éléments de réponse originaux concernant les effets des modifications de températures hivernales sur le fonctionnement photosynthétique des conifères et pourrait être intégré dans un modèle global de simulation des influences des facteurs cli- matiques sur la productivité des peuplements forestiers.

Les travaux de Guehl (1985) et Guehl et al (1985) ayant révélé des fonctionnements photosynthétiques hivernaux notablement différents selon les espèces, il sera nécessaire de déterminer expérimentalement, pour chaque génotype considéré, les fonctions de réponse caractérisant les effets des paramètres climatiques sur l'assimilation de $\mathrm{CO}_{2}$. 


\section{ANNEXE}

\section{MÉTHODE D'ESTIMATION DE LA} DENSITÉ DE FLUX DE RAYONNEMENT SOLAIRE GLOBAL AU NIVEAU DE L'HEURE, EN FONCTION DE LA DURÉE D'INSOLATION JOURNALIĖRE

La procédure d'estimation est effectuée par les opérations suivantes:

1. Estimation du rayonnement solaire global cumulé sur la journée $\left(R_{J}\right)$ avec les formules de régression sulvantes spécifiques de chaque saison

Hiver (déc, janv, fév): $R_{J}=(0,15+0,49$ $\left.\left(F_{\text {INS }}\right)^{1 / 2}\right) R_{E X}$

Printemps (mars, avr, mai) : $R_{J}=(0,21+$ $\left.0,56 F_{\text {INS }}\right) R_{E X}$

Eté (juin, juil, août) : $R_{J}=(0,21+0,54$ $\left.F_{\text {INS }}\right) R_{E X}$

Automne (sept, oct, nov) : $R_{J}=(0,20+$ $\left.0,54 F_{\text {INS }}\right) R_{E X}$

Où $R_{E X}$ est le rayonnement qui serait reçu par une surface horizontale à la limite supérieure de l'atmosphère; $F_{I N S}$ est la fraction d'insolation journalière (durée d'insolation/durée du jour).

2. Estimation du rayonnement solaire reçu sous forme diffuse et cumulté sur la journée $\left(\boldsymbol{R}_{d j}\right)$

$$
\begin{aligned}
& R_{d J}=\left(0,18+0,40 F_{I N S}\right) R_{E X} \text { si } F_{I N S}<0,20 \\
& R_{d J}=0,26 R_{E X} \quad \text { si } 0,20 \leq F_{I N S} \leq 0,75 \\
& R_{d J}=0,53-0,40 F_{I N S} \quad \text { si } F_{I N S}>0,75
\end{aligned}
$$

3. Calcul de la composante verticale du rayonnement solaire direct cumulee sur la journée $\left(\boldsymbol{R}_{D}\right)$

$$
R_{D J}=R_{J}-R_{D J}
$$

4. Calcul de la densité de flux de rayonnement solaire direct horaire $\left(\mathbf{R}_{\mathrm{DH}}\right)$ et de la densité de flux de rayonnement solaire diffus $\left(\mathbf{R}_{\mathrm{dH}}\right)$

$$
R_{D H}=C_{D}(H) R_{D j} /{\stackrel{H C}{\Sigma} C_{D}(H)}_{H}
$$

avec : $C_{D}(H)=\sin \left(H_{S o}\right) \mathrm{e}^{(-0,162 / \sin (s o))}$

où $H_{\text {So }}$ est la hauteur angulaire du soleil au dessus de l'horizon calculée au milieu de l'intervalle horaire $H$ considéré.

Hc

$R_{D H}=C_{d}(H) R_{D j} / \sum_{H I} C_{d}(H)$

$C_{d}(H)$ dépend de la fraction d'insolation $F_{\text {INS }}$ :

$$
\begin{array}{ll}
C_{d}(H)=\left(\sin \left(H_{S o}\right)\right)^{1,172} & \text { si } F_{I N S}<0,1 \\
C_{d}(H)=\left(\sin \left(H_{S o}\right)\right)^{1,08} & \text { si } 0,1 \leq F_{I N S} \leq 0,8 \\
C_{d}(H)=\left(\sin \left(H_{S o}\right)\right)^{0,784} & \text { si } F_{I N S}>0,8
\end{array}
$$

Les coefficients $C_{D}(H)$ et $C_{d}(H)$ représentent respectivement les fonctions de répartition des rayonnements solaire direct et diffus, divisés par leur somme sur l'ensemble des intervalles horaires $H$ compris entre le lever $\left(H_{j}\right)$ et le coucher $\left(H_{\partial}\right)$ du soleil. Le calcul est réalisé selon le temps universel (UTC) pour la délimination des intervalles horaires. 


\section{Calcul de la densité de flux de rayonnement solaire global horaire $\left(\boldsymbol{R}_{H}\right)$}

$$
R_{H}=R_{D H}+R_{d H}
$$

\section{RÉFÉRENCES}

Becker M (1988) Bilan de santé actuel et rétrospectif du sapin (Abies alba Mill) dans les Vosges. Etude écologique et dendrochronologique. Ann Sci For 44, 379-402

Choisnel E (1977) Le bilan d'énergie et le bilan hydrique du sol. Météorologie 6, 103-159

Dickinson RE (1986) How will climate change. The climate system and modelling of future climate. In : The greenhouse effect, climatic change and ecosystems (B Bolin, BR Döös, $\mathrm{J}$ Jäger, RA Warrick eds) Wiley, Chichester, 207-270

Emmingham WH, Waring RH (1977) An index of photosynthesis for comparing forest sites in Western Oregon. Can J For Res 7, 165174

Ericsson A (1978) Seasonal changes in translocation of ${ }^{14} \mathrm{C}$ from different age-classes of needles on 20-year-old Scots pine trees (Pinus silvestris). Physiol Plant 43, 351-358

Ericsson A (1979) Effects of fertilization and irrigation on the seasonal changes of carbohydrate reserves in different age-classes of needle on 20-year-old Scots pine trees (Pinus silvestris). Physiol Plant 45, 270-280

Ericsson A, Persson H (1980) Seasonal changes in starch reserves and growth of fine roots of 20-years-old Scots pines. In : Structure and function of northern coniferous forests - an ecosystem study (Y Persson, ed) Ecol Bull, Stockholm, 32, 239-250

Fry DJ, Phillips IDJ (1977) Photosynthesis of conifers in relation to annual growth cycles and dry matter production. II. Seasonal photosynthetic capacity and mesophyll ultrastuc- ture in Abies grandis, Picea sitchensis, Tsuga heterophylla and Larix leptolepis growing in SW England. Physiol Plant 40, 300-306

Guehl JM (1985) Étude comparée des potentialités hivernales d'assimilation carbonée de trois conifères de la zone tempérée (Pseudotsuga menziesii Mirb, Abies alba Mill et Picea excelsa Link). Ann Sci For 42, 23-38

Guehl JM, de Vitry C, Aussenac G (1985) Photosynthèse hivernale du douglas vert ( $P$ seudotsuga menziesii (Mirb) franco) et du cèdre (Cedrus atlantica Manetti et Cedrus libani Loud). Essai de modélisation à l'échelle du rameau. Oecol Plant 6, 125-146

Krueger KW, Trappe JM (1967) Food reserves and seasonal growth of douglas-fir seedlings. For Sci 13, 192-202

Leverenz J, Öquist G (1987) Quantum yields of photosynthesis at temperatures between -2 and $35^{\circ} \mathrm{C}$ in a cold tolerant $\mathrm{C}_{3}$ plant (Pinus sylvestris) during the course of one year. Plant Cell Environ 10, 287-295

Lundmark T, Hällgren JE, Heden J (1988) Recovery from winter depression of photosynthesis in pine and spruce. Trees 2, 110-114

Pisek A, Kemnitzer R (1968) Der Einfluß von Frost auf die Photosynthese der Weißtanne (Abies alba Mill) Flora Abt B 157, 314-326

Schwarz W (1971) Das Photosynthesevermögen einiger Immergrüner während des Winters und seine Reaktivierungsgeschwindigkeit nach scharfen Frösten. Ber Dtsch Bot Ges Bd 84 (H 10), 585-594

Senser M, Schötz F, Beck E (1975) Seasonal changes in structure and function of spruce chloroplasts. Planta 126, 1-10

Webb WL (1977) Seasonal allocation of photoassimilated carbon in Douglas fir seedlings. Plant Physiol 60, 320-322

Zelawski W, Kucharska J (1967) Winter depression of photosynthetic activity in seedlings of Scots pine (Pinus silvestris L). Photosynthetica 1, 207-213 